\title{
Use of Balloon-Expandable Stents to Treat Experimental Peripheral Pulmonary Artery and Superior Vena Caval Stenosis: Preliminary Experience
}

\author{
Albert P. Rocchini, * Jon N. Meliones, Robert H. Beekman, Catherine Moorehead, and \\ Manette London \\ Division of Pediatric Cardiology, C. S. Mott Children's Hospital, University of Michigan Medical School, \\ Ann Arbor, Michigan, USA
}

SUMMARY. Current therapy of congenital or acquired stenoses of the peripheral pulmonary arteries and superior vena cava are frequently ineffective. This report describes our initial experience with the use of a balloon-expandable stainless steel stent to treat experimentally created branch pulmonary artery and superior vena cava stenosis. Fifteen adult mongrel dogs had surgically created stenoses of either a branch pulmonary artery and/or superior vena cava. A balloon-expandable stainless steel $(0.076 \mathrm{~mm}), 3 \mathrm{~cm}$ long, intravascular stent was used in all animals. Stents were successfully placed in 13 of 15 dogs (nine with branch pulmonary stenosis and four with superior vena caval stenosis) with hemodynamic and angiographic relief of the stenoses in all. In three animals, successful stent placement was not accomplished because the distal right pulmonary artery was found to be totally obstructed in two and in one dog with combined vena cava and pulmonary stenosis the distal right pulmonary artery was so severely stenotic that the stenosis could not be crossed. Repeat catheterization performed 6 months following stent placement documented persistent gradient relief and angiographic evidence of unobstructed flow through the stent without thrombus formation and with patent side branch vessels. Our preliminary results suggests that balloon-expandable stents are a potential therapy for the treatment of branch pulmonary artery and superior vena cava stenoses.

KEY WORDS: Balloon angioplasty-Congenital heart disease

Peripheral pulmonary artery stenosis and superior vena caval stenosis are two lesions in which surgical reconstruction is difficult and sometimes impossible to perform. Although balloon dilation has also been used to successfully treat patients with peripheral pulmonary artery stenosis $[2,4,9,12,13]$ or vena caval stenoses $[5,10,14]$, there are many patients who have had less than satisfactory results. Balloon angioplasty has been reported to be successful in dilating approximately $50 \%$ of patients with branch pulmonary artery stenosis $[2,13]$ and in

\footnotetext{
* Present address: Division of Pediatric Cardiology, Variety Club Children's Hospital, University of Minnesota Medical School, Minneapolis, MN 55455, USA.

Address offprint requests to: Dr. Albert P. Rocchini, Division of Pediatric Cardiology, Variety Club Children's Hospital, University of Minnesota Medical School, Box 94, Minneapolis, MN 55455, USA.
}

approximately $80 \%$ of patients with superior vena caval obstruction [5]. In both of these lesions, although angioplasty can acutely dilate the stenotic vessel to three or four times its original size, the stenoses frequently recur immediately following balloon deflation. The immediate recurrence of obstruction after dilation is thought to be due to the natural elastic recoil of the pulmonary artery and superior vena cava or, in the postoperative cases, due to resilience and resistance of scar tissue [2].

A stainless steel balloon-expandable graft (stent) has recently been developed by Palmaz and coworkers [7, 8]. Both Mullins and coworkers [6] and Benson and coworkers [1] have recently demonstrated the feasibility of placing such stents into the normal pulmonary arteries of dogs and pigs. There is only limited experience with the use of these stents to treat experimentally created stenoses. The purpose of this report is, therefore, to 


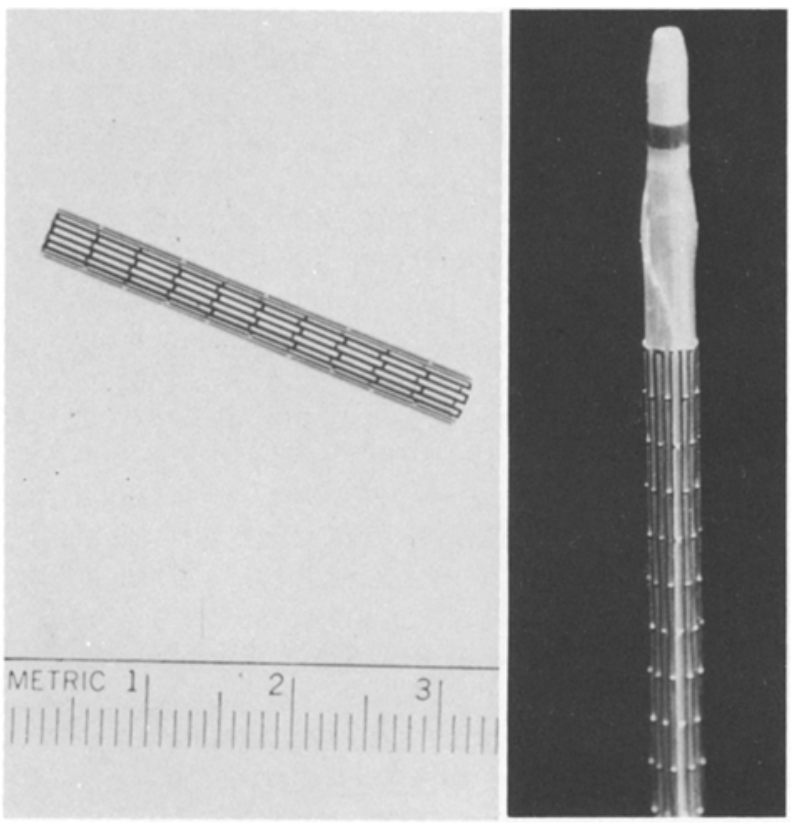

Fig. 1. This is a photograph of an unexpanded stainless steel stent. In the right-hand panel the stent has been placed on a 8$\mathrm{mm}$ balloon angioplasty catheter.

describe our experience with the development of a dog model of peripheral pulmonary artery and/or superior vena caval stenosis and to evaluate the efficacy of relieving the experimentally created stenosis with a balloon-expandable stainless steel stent.

\section{Methods}

\section{Creation of Stenotic Lesion}

Peripheral pulmonary artery stenosis and/or superior vena cava stenosis was created in 15 adult mongrel dogs [11], weighing 19.2 $\pm 2.3 \mathrm{~kg}$. All dogs were anesthetized with halothane anesthesia. A thoracotomy was performed and the left or right pulmonary artery and/or superior vena cava isolated. Two pieces of 2-0 vicryl suture (three loops each) were placed around the pulmonary artery $1-2 \mathrm{~cm}$ from its origin and/or placed around the cava $1-2 \mathrm{~cm}$ from its junction with the right atrium. The sutures were tightened so that the pulmonary artery and/or vena cava was narrowed by $30-40 \%$. All dogs received intramuscular antibiotics for 3 days after surgery to prevent infection and morphine sulfate as necessary for pain. Isolated right pulmonary artery stenosis was created in four dogs, isolated left pulmonary artery stenosis in seven, isolated superior vena cava stenosis in three, and combined right pulmonary artery stenosis and superior vena caval stenosis in one.

\section{Stent Placement}

Two to three months after creation of the stenosis all dogs underwent cardiac catheterization under suritol anesthesia. The right heart catheterization was performed percutaneously from the right femoral area. After hemodynamic (superior vena cava, right atrial, right ventricular, main pulmonary artery, proximal left pulmonary artery, and distal left pulmonary artery pressures were measured) and angiographic (right or left pulmonary artery and/or superior vena cava angiograms) assessment of the stenosis, stent placement was attempted. A no. 7F end-hole catheter was passed into the distal pulmonary artery or vena cava. A 0.038 -inch Teflon-coated $250-\mathrm{cm}$ exchange guidewire was advanced into the distal vessel. The end-hole catheter was removed, leaving the wire fixed in the vessel, and a no. $12 \mathrm{~F}$ (for the pulmonary arteries) or $16 \mathrm{~F}$ (for the superior vena cava) long sheath and dilator were introduced over the guidewire into the vessel beyond the stenosis. Although the $8-\mathrm{mm}$ angioplasty balloon with stent could fit through a $10 \mathrm{~F}$ sheath, because of the canine anatomy a $12 \mathrm{~F}$ sheath provided each movement of the balloon and stent across the tricuspid valve, pulmonary valve, and right pulmonary artery. Despite the discrepancy between sheath and catheter, bleeding from the sheath was minimal and a back-flow device was not necessary. A stainless steel stent, which was $0.076 \mathrm{~mm}$ in thickness, $3 \mathrm{~cm}$ in length, and $3.7 \mathrm{~mm}$ in diameter before expansion (provided by Johnson and Johnson Inc.), was placed on an 8-mm diameter (for pulmonary arteries) or 18-mm diameter (for the superior vena cava) balloon angioplasty catheter (Fig. 1). The size of the angioplasty catheter was chosen to be $\sim 1 \mathrm{~mm}$ larger than the normal vessel diameter. The long dilator was removed from the sheath and the stent-mounted balloon angioplasty catheter was advanced through the long sheath into the distal pulmonary artery or vena cava. After the angioplasty catheter was positioned across the stenosis, the sheath was withdrawn off the proximal end of the angioplasty catheter into the main pulmonary artery or right ventricle (for pulmonary arteries) or into the right atrium (for the superior vena cava). The balloon was then expanded to 4-6 atm of pressure. The balloon was deflated and then exchanged for an end-hole catheter leaving the expanded stent across the area of stenosis. Repeat hemodynamics and angiography were performed. The catheter was removed and the groin held until hemostasis was obtained. To prevent thrombus formation in the long sheath, the dogs received $50 \mathrm{U} / \mathrm{kg}$ heparin during the catheterization. The dogs received 3 days of antibiotic after the catheterization and morphine sulfate as necessary for pain. The dogs received no anticoagulation or antiplatelet agents. The dogs were recatheterized at 3 and 6 months following placement of the stent. At these catheterizations hemodynamic and angiographic assessment of the stent was made. After the stent had been in place for 6 months, the dogs were killed for pathological assessment of the stent and vessel.

\section{Statistical Analysis}

All data are presented at the mean \pm standard error (SE). To assess changes in the gradient across stenoses over time, a repeated measures analysis of variance was used.

\section{Results}

Dilatable peripheral pulmonary artery stenosis or superior vena caval stenosis was produced in 13 of 15 dogs (peripheral pulmonary artery stenosis in nine and superior vena caval stenosis in four). In 
the two remaining dogs the distal right pulmonary artery was found to be totally obstructed and in the dog with combined vena cava and pulmonary stenosis the distal right pulmonary artery stenosis was so severe that neither a catheter nor guidewire could be advanced across the stenosis.

Prior to attempted stent placement, the peak systolic gradient across the stenoses averaged $32 \pm$
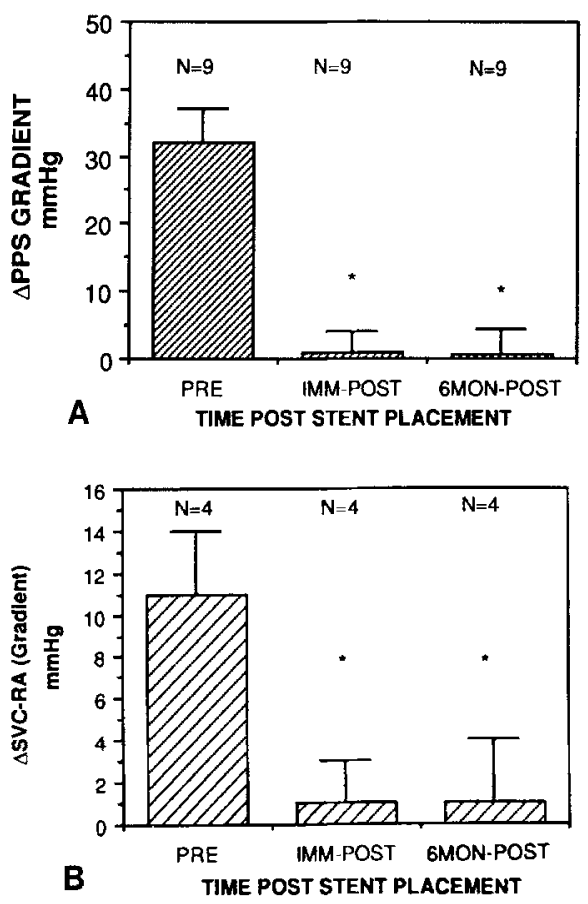

Fig. 2. The serial peak systolic gradients across the branch pulmonary arteries are depicted in panel $\mathbf{A}$. The stent placement resulted in an immediate improvement of the gradient, which was maintained for the 6 months of follow-up. Panel $\mathbf{B}$ depicts the serial mean gradients across the experimentally created superior vena cava stenosis. As with the branch pulmonary arteries, the stent placement resulted in a complete elimination of the stenosis. Statistical analysis was performed using repeated measures analysis of variance $(*, p<0.05)$.
$8 \mathrm{mmHg}$ for 9 of 12 dogs with branch pulmonary artery stenosis, and the mean gradient in the four dogs with superior caval stenosis averaged $11 \pm 4$ $\mathrm{mmHg}$. Successful stent placement was accomplished in all 13 dogs in which the stenotic site could be crossed with the $7 \mathrm{~F}$ wedge catheter. In all cases the stent abolished the gradient across the stenosis (Fig. 2). In all but one dog, there were no significant complications associated with stent placement. In this dog in which stent placement was attempted, two of the stents inadvertently embolized from the angioplasty catheter. In this animal the stent dislodgements occurred while trying to advance the angioplasty catheter and stent across the tricusipid valve without the use of a long sheath. When a long sheath was used to deliver the stent in this and the remaining dogs, no inadvertent embolization occurred.

At follow-up catheterization, 3 and 6 months poststent placement, all stents were patent without evidence of thrombosis (Figs. 3 and 4). In the five dogs with distal pulmonary artery stent placement, multiple branch pulmonary arteries were crossed by the stent and, at follow-up catheterization, angiography demonstrated that these branch pulmonary vessels remained patent (Fig. 5). Finally, there was no evidence for either late stent migration to a more distal position in the pulmonary artery or for the development of aneurysms.

\section{Pathology}

The stents in the pulmonary arteries and superior vena cava were covered with neointima. However, in the three dogs with proximal left pulmonary artery stenosis, the small portion of the stent, which extended into the main pulmonary artery, was the only portion of the stent not covered by a thin layer of neointima. All stents were patent and there was

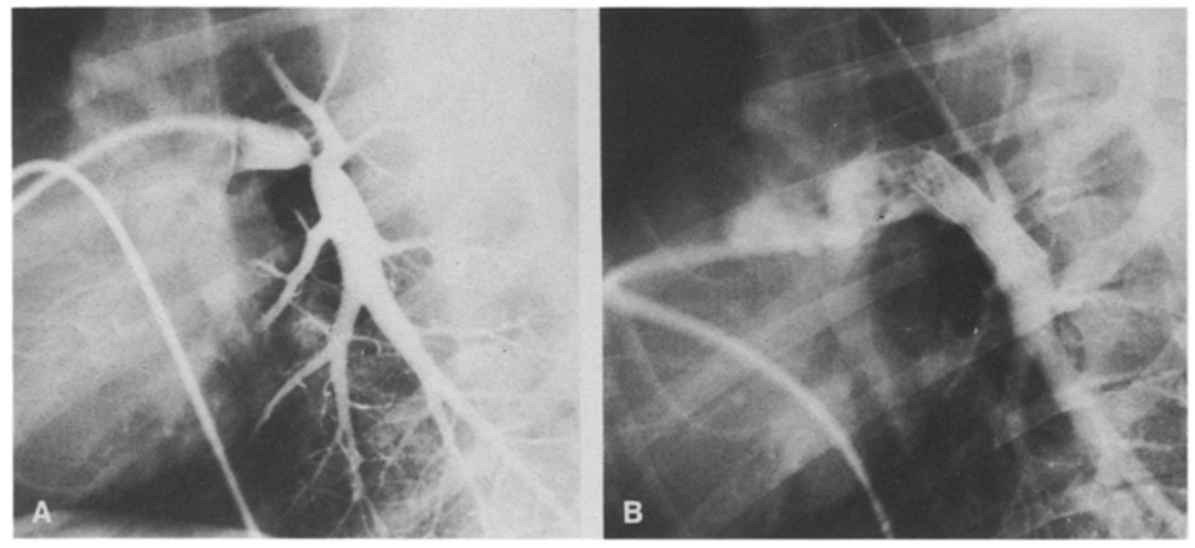

Fig. 3. A series of angiogram before $(\mathbf{A})$ and 6 months after (B) placement of a stent in a branch pulmonary. In panel $\mathbf{A}$ the discrete stenosis of the proximal left pulmonary artery is visualized. Panel $\mathbf{B}$ angiographically demonstrates that the stent and pulmonary artery are widely patent 6 months after initial placement. 

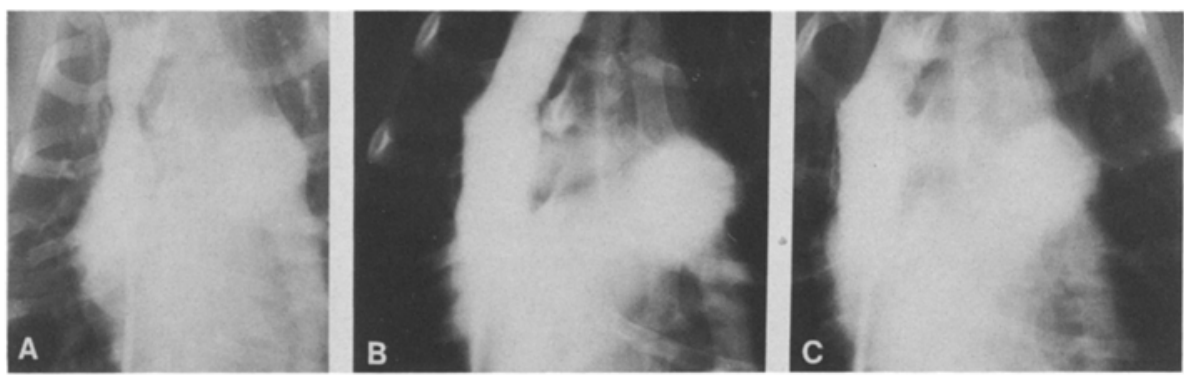

Fig. 4. A series of angiograms before (A), immediately after (B), and 6 months postplacement of a stent in the superior vena cava (C). In panel $\mathbf{A}$ the discrete stenosis of the superior vena cava is visualized. Panels $\mathbf{B}$ and $\mathbf{C}$ demonstrate that the vena cava and stent are widely patent immediately postplacement $(\mathbf{B})$ and 6 months postplacement $(\mathbf{C})$.

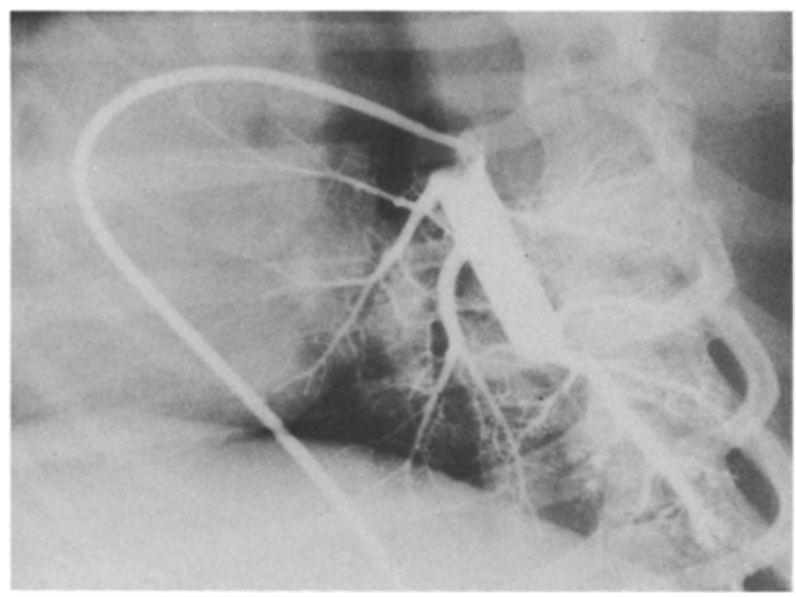

Fig. 5. An angiogram of a distally placed stent 6 months after initial placement demonstrates that the branch pulmonary arteries that were crossed by the stent are widely patent. Although there appears to be a narrowing of the distal pulmonary artery, no hemodynamic gradient was present.

no evidence of thrombosis in the stent or distal to the stent. In those animals in which the stents crossed side branches of the pulmonary artery, these side branches were patent at postmortem examination. No microscopic analysis was performed.

\section{Discussion}

Peripheral pulmonary artery stenosis and superior vena caval stenoses are two lesions in which both surgical reconstruction and balloon angioplasty are difficult and frequently impossible to successfully perform $[2,4,5,14,15]$. The current study demonstrates that a stainless steel balloon-expandable stent can provide long-term effective relief of both experimentally created peripheral pulmonary artery stenosis and superior vena caval stenosis. Our results are in agreement with the work of Mullins and coworkers [6] and Benson et al. [1]. Mullins successfully implanted 11 of 13 stents in normal pul- monary arteries, and 11 of 14 were implanted in normal nonstenotic tributaries of precava or postcava. Some of their dogs were recatheterized at intervals ranging from 56-270 days. Twelve stents were patent and nonobstructive, two were malposed, and one was obstructive. When the animals were killed, the stents were evaluated pathologically and were found to be completely covered with neointima and patent without any evidence of thrombus formation. Other than the current report, there has been limited animal experience with the use of stents to treat experimentally produced stenoses of either the peripheral pulmonary arteries or systemic veins. Mullins and coworkers [6] describes dilating one experimentally created peripheral pulmonary artery narrowing, and Benson et al. [1] has described in an abstract the successful use of stents to dilate surgically created stenoses in the pulmonary arteries of pigs. Thus, based on our work and these other reports, it appears that stainless steel balloon-expandable stents should be ideal for treating subjects with refractory pulmonary artery or vena caval stenosis.

However, before thes stents can be widely recommended for children and infants, a number of other questions need to be addressed. Children frequently will require placement of a stent at a young age prior to achieving full growth. Since these stents are made of stainless steel, they will not grow with the patient and stenosis may therefore recur. Thus, the question of whether these stents can be progressively expanded months or years after initial placement needs to be addressed before they can be used in the young patient. Vick and coworkers [13] have shown that stents placed in the descending aorta of normal juvenile minipigs could be redilated 6 months after initial placement without damage to the aortic wall or stent. Further animal studies will be necessary to establish that balloon dilatable stents can also be redilated when positioned in a branch pulmonary artery or vena cava. A second question that needs to be addressed relates to the ideal length of stent that should be used. As was demonstrated in our dogs with proximally placed 
stenoses, the use of $3-\mathrm{cm}$ stent resulted in a portion of the stent extending into the main pulmonary artery. For these proximal discrete stenoses, the use of a shorter stent length would appear to be more appropriate. In many cases of pulmonary artery branch stenosis, long segment stenosis or multiple stenoses are present. In these situations two or more stents could be implanted in series as has been done in systemic peripheral arterial and coronary artery disease [3]. Experimental studies will need to document that implantation of stents in series in the pulmonary artery will preserve the curvature of the pulmonary vessel without damaging the vessel. Finally, although we and others $[3,7,8]$ have documented that these stents remain patent without local or distal thrombosis formation for up to 6 months, we do not known if thrombi will develop after longer periods of implantation, especially when the stent is placed in a systemic vein.

In addition to using these balloon dilatable stainless steel stents to treat pulmonary artery and vena cava stenosis, these stents may also be useful in treating other forms of congenital or surgically acquired stenoses, such as pulmonary venous stenosis or even coarctation of the aorta.

In summary, we have demonstrated that the balloon-expandable stainless steel stent appears to be an extremely promising new technique for the dilation of refractory pulmonary artery and vena caval stenosis. Therefore we, believe, based on the current report and the work of others, that clinical trials of stent placement in adolescents with refractory pulmonary artery or vena cava stenosis are warranted.

Acknowledgments. The authors which to express their thanks to Johnson and Johnson Inc. Warren, NJ, USA) for providing us with the stents.

This work was supported in part by a grant from the Michigan Heart Association.

\section{References}

1. Benson LN, Hamilton F, Dasmahapatra HK, Coles JG (1988) Implantable stent dilation of the pulmonary artery: Early experience [abstract]. Circulation 78(Suppl II): II-100
2. Kan JS, Marvin WJ, Bass JL, et al. (1990) Balloon angioplasty-branch pulmonary artery stenosis: Results from the valvuloplasty and angioplasty of congenital anomalies registry. Am J Cardiol 65:798-801

3. Levine MJ, Leonard BM, Burke JA, Nash ID, Safian RD, Diver DJ, Baim DS (1990) Clinical and angiographic resuits of balloon-expandable intracoronary stents in right coronary artery stenosis. J Am coll Cardiol 16:332-339

4. Lock JE, Castaneda-Zuniga WR, Furhman BP, Bass JL (1983) Balloon dilation angioplasty of hypoplastic and stenotic pulmonary arteries. Circulation 67:962-967

5. Mullins CE, Latson LA, Neches WH, Colvin EV, Kan J (1990) Balloon dilation of miscellaneous lesions; Results of valvuloplasty and angioplasty of congenital anomalies registry. Am J Cardiol 65:802-803

6. Mullins CE, O'Laughlin MP, Vick GW III, et al. (1988) Implantation of balloon-expandable intravascular grafts by catheterization in pulmonary arteries and systemic veins. Circulation 77:188

7. Palmaz JC, Sibbitt RR, Tio Fo, et al. (1986) Expandable intraluminal vascular graft: A feasibility study. Surgery 99:199

8. Palmaz JC, Windeler SA, Reuter SR, et al. (1987) Expandable intrahepatic portacaval shunt stents: Early experience in the dog. Am J Radiol 145:821

9. Ring JC, Bass JL, Marvin W, Furhman BP, Kulek JJ, Foker JE, Lock JE (1985) Management of congenital stenosis of a branch pulmonary artery with balloon dilation angioplasty. Report of 52 procedures. I Thorac Cardiovasc Surg 90:35

10. Rocchini AP, Cho KJ, Byrum C, Heidelberger K (1982) Transluminal angioplasty of superior vena caval obstruction in a 15 month old child. Chest 82:506

11. Rocchini AP, Gundry SR, Beekman RH, et al. (1988) A reversible pulmonary artery band: Preliminary experience. $J$ Am Coll Cardiol 11:172

12. Rocchini AP, Kveselis DA, Crowley D, Dick M, Rosenthal A (1984) Use of balloon angioplasty to treat peripheral pulmonary stenosis. Am J Cardiol 54:1069

13. Rothman A, Perry SB, Keane JF, Lock JE (1990) Early results and follow-up of balloon angioplasty for branch pulmonary artery stenosis. $J$ Am Coll Cardiol 16:1109-1117

14. Vick GW III, O'Laughlin M, Myers T, Nakatani T, Palmaz J, Schatz R, Morrow WR, Mullins C (1989) Evaluation of aortic implantation and redilation of balloon expandable intravascular stents in juvenile minipigs [abstract]. $J \mathrm{Am}$ Coll Cardiol 13: 223A

15. Waldman JD, Waldman J, Jones MC (1983) Failure of balloon dilation in mid cavitary obstruction of the systemic venous atrium after Mustard operation. Pediatr Cardiol 4:151154

16. Wilson JM, Mack JW, Turley K, Ebert PA (1978) Persistent stenosis and deformity of the right pulmonary artery after correction of the Waterston anastomosis. I Thorac Cardiovasc Surg 82:169 